\title{
Trigeminal Neuralgia: Case Report and Treatment Modalities
}

\section{Nilotpol Kashyap*, Abhinab Neog, Kangkan Roy, Santanu Borah, Monalisa Borah and Alina Aryal}

Pedodontics and Preventive Dentistry, Chitwan Medical College Nepal, Nepal

*Corresponding Author: Nilotpol Kashyap, Pedodontics And Preventive Dentistry, Chitwan Medical College Nepal, Nepal.

Received: September 09, 2019; Published: September 23, 2019

DOI: $10.31080 / A S D S .2019 .03 .0647$

\section{Abstract}

The International Association for the Study of Pain (IASP) definition of trigeminal neuralgia (TN) is "sudden usually unilateral severe brief stabbing recurrent pains in the distribution of one or more branches of the fifth cranial nerve" [1]. Nicolaus Andre had used the term 'tic douloureux' [2].

It is a well-recognized disorder that most commonly occurs in people over 50 years of age and slightly more commonly in women than in men.

It is a very peculiar disease. The pain is paralytic and very severe. It can be triggered by a light cutaneous stimulus on a very localized spot on the face (the so called "trigger zone") [3].

Keywords: Trigeminal Neuralgia; Pain

\section{Epidemiology}

Few data are available. Incidence rate of $\mathrm{TN}$ is about 3 to 5 cases/year/100.000 persons [4,5]. Prevalence has been estimated at 107.5 men and 200.2 women/1 million population [6]. Risk factors have been investigated: multiple sclerosis is well known but additional risk factors are not confirmed [7].

\section{Etiology}

Trigeminal neuralgia is an idiopathic disorder, but several theories of neuropathological processes attempt to explain the symptoms. One theory is compression of the trigeminal root adjacent to the pons, causing demyelination of the nerve root. This may be caused by tortuous blood vessels in the posterior fossa, tumors or arteriovenous malformations. However, these structural lesions are not found in all patients with TN. Other theories suggest that TN is a symptom of a central nerve disease or a disease of the trigeminal vascular system involving dysfunctional interplay between a trigeminal sensory plexus and blood vessels located in the pia and durometer [8].

\section{Clinical features}

- Clinical features of pain in TN are [9]

- Character-Shooting, stabbing, sharp, electric

- Site/radiation- Trigeminal distribution only, intraoral and extraoral,

- Severity- Moderate to severe

- Duration-1-60 seconds refractory period

- Periodicity- Rapid onset and termination, complete periods of remission weeks to months

- $\quad$ Provoking factors- Light touch, washing the face, brushing teeth, shaving, applying make-up, going out in cold wind, vibrations from walking

- Relieving factors- Keeping still, drugs

- Associated factors- Local anaesthesia placed in trigger area relieves pain, severe depression and weight loss (Table 1).

\begin{tabular}{|l|c|c|c|}
\hline \multicolumn{1}{|c|}{ Condition } & Location of pain & Duration of pain or attack & Shooting pain or symptoms \\
\hline Cluster head ache & Retrobulbar, cheek, chin & 20 min-hours & Only superimposed on deep dull pain \\
\hline SUNCT* & Forehead, retrobulbar & 5 seconds to several minutes & Yes \\
\hline CPH $^{* *}$ & Forehead, retrobulbar & $2-45$ minutes & Yes \\
\hline Cracked tooth syndrome & Upper jaw, lower jaw, & Seconds & Yes \\
\hline Jabs and jolts syndrome & anywhere in head & Seconds & Superimposed background pain \\
\hline Post-herpetic neuralgia & Forehead, eye, check (rarely) & Continuous & None \\
\hline Giant cell arteritis & Forehead, neck, temple & Continuous \\
\hline
\end{tabular}

Table 1: *SUNCT- Short-lasting unilateral neuralgiform headache with conjunctival injection and tearing. ${ }^{* *} \mathrm{CPH}$-Chronic paroxysmal hemicranias. 
Differential diagnosis [2]

Investigations

Patient history is the most useful tool for diagnosing classical TN. However, recent advances in neuroimaging have improved the ability to diagnose symptomatic TN [9].

\section{Treatment options}

As in other fields of medicine, a mechanism based treatment should be favoured. The neurosurgeon provides pain relief by alleviating an "offending contact" with the nerve.

History of TN is however characterized by many symptombased treatments: peripheral nerve section or neurolytic blocs (alcohol), a variety of physical agents have been used to provoke a (hopefully) minimal damage to the trigeminal ganglion or root and surgical rhizotomy has also been proposed [10].

\section{Medical treatment}

Carbamazepine (CBZ) remains the drug of first choice. Treatment begins with 100 to $200 \mathrm{mg}$ two or three times daily. Doses should be increased very progressively and titrated to the severity of the patient's pain. Serum level is a useful way of monitoring treatment ( 6 to $12.5 \mu \mathrm{g} / \mathrm{ml}$ ). In some cases a maintenance dosage of $200 \mathrm{mg}$ or $400 \mathrm{mg}$ per day is sufficient to keep the patient painfree. With appropriate adjustments of dosage, pain can be controlled initially in about $75 \%$ of patients [11].

If paroxysms of pain still occur with therapeutic blood levels, another drug should be added: baclofen or phenytoin. Other drugs, including sodium valproate, gabapentin, lamotrigine and clonazepam, have been tried but formal conclusive studies are still lacking [11].

\section{Surgical treatment}

If a patient does not obtain relief from pain with medical treatment, some form of surgery will be proposed. It is estimated that up to $50 \%$ of the patients will sooner or later be in that situation [12]. Historically, many operations have been proposed, more or less invasive. Among invasive procedures, we find operations aimed at lesioning nerve fibers or ganglion cells (peripheral nerve section, ganglionectomy, rhizotomy), and a non-destructive operation aimed at relieving the nerve root from an offending contact ("decompression"). In order to reduce the risk, percutaneous approaches have been developed, using chemical or physical agents to impair transmission of impulses in the trigeminal pathway while avoiding major loss of function. Among these percutaneous procedures we find differential thermal rhizotomy, glycerol rhizotomy and compression of Gasser ganglion by a balloon (so-called “microcompression") [11].

\section{Case Report}

A 51 year old female patient (Figure 1) reported to the department with the chief complaint of pain in respect to upper left part of face since last two and a half months.

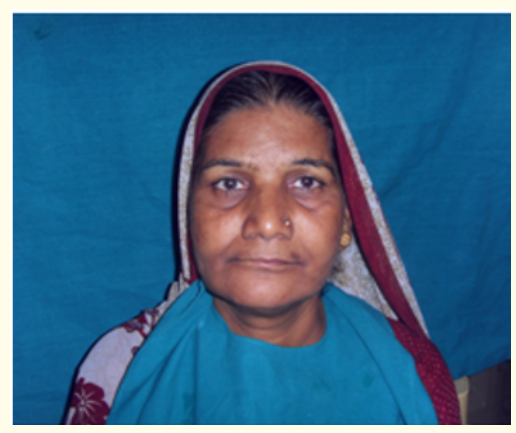

Figure 1

Patient gave the history of severe and excruciating paroxysmal episodes of pain, multiple episodes per day. Duration of each episode was around two and a half minutes approximately. Pain radiated to the head (parietal region of the same side). Pain aggravated on washing face or in contact of clothes or when exposed to cold breeze. No relevant medical history was revealed.

Patient gave the dental history of extraction in relation to the upper arch 35 years back and was wearing a denture since then.

Local examination revealed non tender lymph nodes and TMJ also showed neither clicking nor deviation.

Extraoral examination did not reveal any significant findings.

Intraoral examination revealed missing teeth in relation to both upper and lower arch (Figure 2 and 3).

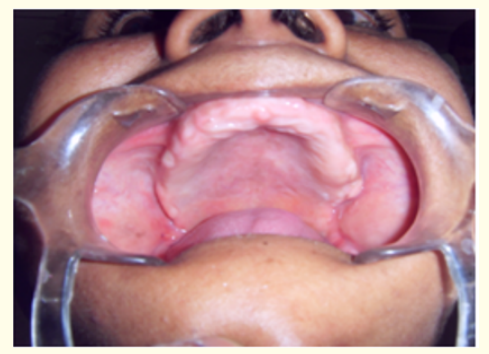

Figure 2

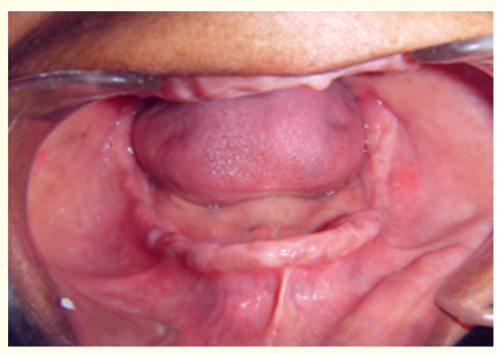

Figure 3 
On the basis of history and clinical findings, the provisional diagnosis made was Trigeminal neuralgia.

\section{Investigations advised}

Patient was having her own Panoramic radiograph (Figure 4), advised earlier by a clinician which showed no evidence of any bony pathology.

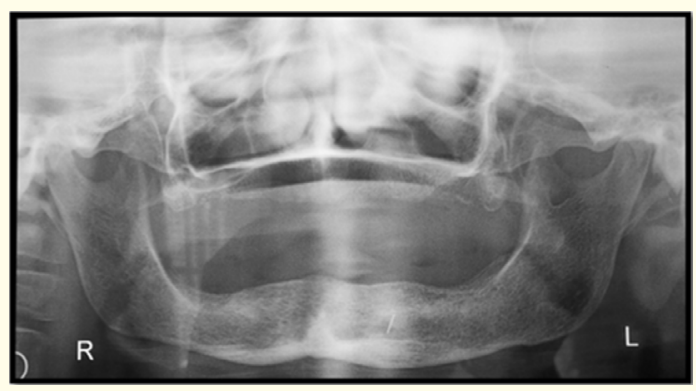

Figure 4

A consultation was taken with the department of Neurosurgery.

Treatment advised was

- Tab. Tegrectol (200 mg) 1-1-1 x 3 days

- Cap. Omez (20 mg) 0-1-0 x 3 days

- Patient was recalled after 3 days for follow up.

- On follow up after 3 days, patient reported that pain was reduced and symptoms were relieved after taking medication.

- $\quad$ Patient got relief while taking medication for 4 - 5 months and then pain started again after some time.

- Peripheral neurectomy was done in relation to infraorbital nerve.

\section{Discussion}

Trigeminal neuralgia is a clinical diagnosis. The key feature is a sudden and severe lancinating pain, which usually lasts from a few seconds to two minutes, within the trigeminal nerve distribution, typically the maxillary or mandibular branches. The pain is often evoked by trivial stimulation of appropriately named "trigger zones".

\section{Conclusion}

Trigeminal neuralgia is the most common neurologic cause of facial pain. The first step is to make the correct diagnosis. Initial therapy should be low-dose carbamazepine, with upward titration of the dose to relief of pain.

Baclofen is a good choice for second-line therapy. Most patients will respond to anticonvulsant therapy, but its effectiveness decreases with time.

Newer medications have shown promise, but none has been shown to be more effective than carbamazepine and need not be used for first line therapy. Effective surgical options are available for cases that do not respond to medical treatment.

\section{Bibliography}

1. Cruccu G., et al. "AAN-EFNS guidelines on trigeminal nurelgia management". European Journal of Neurology 15.10 (2008): 1013-1028.

2. Nurmikko TJ and Eldridge PR. "Trigeminal neuralgia- pathophysiology, diagnosis and current treatment". British Journal of Anaesthesia 87 (2001): 117-132.

3. Klieb HBE and Freeman BV. "Trigeminal Neuralgia Caused by Intracranial Epidermoid Tumour: Report of a Case”. Journal of the Canadian Dental Association 74.1 (2008): 63-65.

4. Katusik S., et al. "Incidence and clinical features of trigeminal neuralgia: Rochester Minnesota, 1945-1984". Annals of Neurology 27.1 (1990): 89-95.

5. Rothman KJ and Monson RR. "Epidemiology of trigeminal neuralgia”. Journal of Chronic Diseases 26 (1973): 3-12.

6. Penman J. "Trigeminal neuralgia". In: Handbook of Clinical Neurology. Vinken PJ, Bruyn GW (eds.). Amsterdam, North Holland 5 (1968): 296-322.

7. Kitt CA., et al. "Trigeminal neuralgia: opportunities for research and treatment". Pain 85.1-2 (2000): 3-7.

8. Delzell J E and Grelle AR. "Trigeminal Neuralgia New Treatment Options for a Well-known Cause of Facial Pain" Archives Of Family Medicine 8.3 (1999): 264-268.

9. Zakrzewska J M and McMillan R. "Trigeminal neuralgia: the diagnosis and management of this excruciating and poorly understood facial pain". Postgraduate Medical Journal 87.1028 (2011): 410-416.

10. Gybels JM and Sweet WH: "The trigeminal nerve". In: Neurosurgical treatment of persistent pain. Karger (1989): 10-69.

11. Joffroy A., et al. "Trigeminal neuralgia Pathophysiology and treatment". Acta Neurologica Belgica 101.1 (2001): 20-25.

12. Taylor J C. "Long term treatment of trigeminal neuralgia with carbamazepine”. Postgraduate Medical Journal 57.663 (1981): 16-18.

Volume 3 Issue 10 October 2019

(C) All rights are reserved by Nilotpol Kashyap., et al. 